\title{
Decreased Expression of V-Set and Immunoglobulin Domain Containing 1 (VSIG1) Is Associated With Poor Prognosis in Primary Gastric Cancer
}

\author{
YIBING CHEN, MD, PhD, ${ }^{1}$ KE PAN, PhD, ${ }^{1}$ SHENGPING LI, MD, PhD, ${ }^{2}$ JIANCHUAN XIA, MD, PhD, ${ }^{1 *}$ \\ WEI WANG, MD, PhD, ${ }^{3}$ JUGAO CHEN, MD, PhD, ${ }^{1}$ JINGJING ZHAO, MD, PhD, ${ }^{1}$ LIN LÜ, MD, PhD, \\ DANDAN WANG, PhD, ${ }^{1}$ QIUZHONG PAN, MD, ${ }^{1}$ QIJING WANG, MD, ${ }^{1}$ YONGQIANG LI, MD, \\ JIA HE, MD, ${ }^{1}$ AND QIAO LI, PhD ${ }^{4 * *}$ \\ ${ }^{1}$ State Key Laboratory of Oncology in Southern China and Department of Experimental Research, Sun Yat-Sen University Cancer Center, \\ Guangzhou, China \\ ${ }^{2}$ Department of Hepatobiliary Surgery, Sun Yat-Sen University Cancer Center, Guangzhou, China \\ ${ }^{3}$ Department of Gastric and Pancreatic Surgery, Sun Yat-Sen University Cancer Center, Guangzhou, China \\ ${ }^{4}$ Department of Surgery, University of Michigan Medical Center, Ann Arbor, Michigan
}

\begin{abstract}
Background: To date, the significance of altered expression of V-set and immunoglobulin domain containing 1 (VSIG1) in gastric cancer has not yet been elucidated.

Methods: We examined VSIG1 expression in 30 paired gastric cancer tissues and noncancerous gastric mucosa as well as in 5 gastric cancer cell lines by real-time PCR and Western blotting. In addition, we analyzed VSIG1 expression in 232 gastric adenocarcinoma samples by immunohistochemistry.

Results: VSIG1 expression was significantly reduced at both the mRNA and protein levels in gastric cancer tissues. Immunohistochemistry revealed that VSIG1 expression was completely lost in 126 out of the $232(54.3 \%)$ patient samples and remarkably reduced in another 106 $(45.7 \%)$ patients. Negative VSIG1 expression was significantly correlated with tumor size $(P=0.007)$, T $(P=0.023)$, and $\mathrm{M}$ stage $(P=0.037)$. Importantly, loss of VSIG1 expression was significantly correlated with poor overall survival $(\mathrm{OS}, P<0.001)$ and disease-free survival (DFS, $P=0.006$ ) in gastric cancer patients. Cox regression analyses showed that VSIG1 expression was an independent predictor of OS $(P=0.002)$ and DFS $(P=0.039)$.

Conclusions: Our findings suggest that silencing VSIG1 may play an important role in gastric carcinogenesis and that VSIG1 may serve as a prognostic marker as well as a potential therapeutic target for gastric cancer.
\end{abstract}

J. Surg. Oncol. 2012;106:286-293. (C) 2011 Wiley Periodicals, Inc.

\section{KeY Words: VSIG1; gastric cancer; real-time quantitative PCR; Western blotting; immunohistochemistry; Cox regression}

\section{INTRODUCTION}

Gastric cancer is the fourth most common malignancy in the world and the second leading cause of cancer-related death in humans [1]. Nearly one million new gastric cancer cases are diagnosed each year worldwide, half of which are in Eastern Asia. It is generally thought that gastric cancer results from the combinatorial effects of environmental factors and the accumulation of genetic and epigenetic disorders. Most gastric cancers emerge after a long period of chronic atrophic gastritis and intestinal metaplasia, with the single most common cause being Helicobactor pylori infection [2]. In addition, the consumption of grilled and $N$-nitrosamine-enriched foods is also considered to be highly correlated with gastric carcinogenesis. When clinically diagnosed, a significant number of gastric cancer patients are beyond the limits of curative resection [3]. Understanding the molecular pathways involved in gastric carcinogenesis will be critical for the improvement of diagnosis and therapy of gastric cancer.

Aberrant gene expression, including the activation of oncogenes and the inactivation of tumor suppressor genes, plays an important role in the initiation and progression of gastric cancer [4]. Thousands of genes differentially expressed between normal gastric mucosa and cancerous tissues, including those that mediate cell adhesion, have been identified using high-throughput microarray-based expression profiling [5,6]. Cell adhesion molecules participate in numerous cell
Abbreviations: AJCC, American Joint Committee on Cancer; CAR, Coxsakievirus and adenovirus receptor; $\mathrm{CDH} 1$, cadherin 1, type 1, E-cadherin (epithelial); DAB, 3,3'-diaminobenzidine; DFS, disease-free survival; GAPDH, glyceraldehyde-3-phosphate dehydrogenase; HE, hematoxylineosin; JAM, junctional adhesion molecule; JGCA, Japanese Gastric Cancer Association; MAGUK, membrane-associated guanylate kinase; MAGI, MAGUK inverted; OS, overall survival; PBS, phosphate-buffered saline; PCR, polymerase chain reaction; UICC, Union for International Cancer Control; VSIG1, V-set and immunoglobulin domain containing 1.

Grant sponsor: National Natural Foundation of China; Grant number: 30973398; Grant sponsor: Gillson Longenbaugh Foundation.

Conflict of interest: none.

*Correspondence to: Dr. Jianchuan Xia, MD, PhD, State Key Laboratory of Oncology in Southern China and Department of Experimental Research, Sun Yat-Sen University Cancer Center, 651 Dongfeng Road East, Guangzhou 510060, China. Fax: +86-20-87343392.

E-mail: xiajch@mail.sysu.edu.cn

**Correspondence to: Qiao Li, PhD, Department of Surgery, University of Michigan Medical Center, 1520A MSRB-1, Ann Arbor, MI 481090666. Fax: 734-763-4135. E-mail: qiaoli@umich.edu

Received 1 July 2011; Accepted 28 October 2011

DOI 10.1002/jso.22150

Published online 17 November 2011 in Wiley Online Library (wileyonlinelibrary.com). 
functions, for example, signal transduction, cell growth, differentiation, cell motility, and immune function [7]. In fact, changes in the expression and function of cell adhesion molecules have been implicated in all steps of tumor development and progression. These disorders cause disruption of cell-cell or cell-extracellular matrix interactions, which significantly contribute to the uncontrolled proliferation, progression, and metastasis of cancer cells. One of the classical examples is E-cadherin, a calcium-dependent cell adhesion glycoprotein encoded by the $C D H 1$ gene, which has been shown to be exclusively expressed in epithelia and frequently silenced in carcinoma, including gastric cancer [8-10]. In contrast, overexpression of integrin $\beta 3$ is correlated with poor prognosis of gastric cancer [11].

Increasing attention has recently been paid to the role of tight junctions in carcinogenesis. Tight junctions are a characteristic feature of epithelial cells and are constructed from a complex array of integral and peripheral proteins [12]. The former include occludin, tricellulin, claudins, junctional adhesion molecules (JAMs) and Crumbs, whereas the latter comprise a wide spectrum of proteins, including the membrane-associated guanylate kinase (MAGUK) family, MAGUK inverted (MAGI) proteins, cingulin and symplekin. Two major functions defined for tight junctions are the regulation of paracellular permeability and the maintenance of cell polarity. The development of epithelial tumors is associated with a loss of cell polarity and disturbances in the structure and function of tight junctions. The down-regulation of JAM-A has been reported to be involved in the progression of clear cell renal cell carcinoma [13] and breast cancer cells [14], whereas JAM-C promotes tumor growth and angiogenesis [15]. In gastric cancer, decreased expression of claudins- 1,4 , and 11 is associated with a more malignant cancer phenotype [16-18], while the expression of claudin-7 is increased in intestinal-type gastric cancer [19] and may cause epithelial dysfunction [20]. Coxsakievirus and adenovirus receptor (CAR), another member of the JAM family, has been reported to suppress the proliferation, migration, and invasion of gastric cancer cells, and the loss of CAR predicts poor prognosis in gastric cancer [21]. Overall, these observations underline the complex roles of tight junction proteins in gastric cancer.

V-set and immunoglobulin domain containing 1 (VSIG1), a newly discovered member of the JAM family, is widely expressed in gastric epithelia [22]. A recent microarray study showed that VSIG1 expression was reduced in both Japanese and Finnish gastric cancer tissues [23]. However, the clinical significance of such differential expression and the function of the VSIG1 protein have not yet been defined. In this study, we evaluated the expression of VSIG1 using quantitative real-time PCR, Western blotting, and immunohistochemistry. We determined its correlation with clinicopathological parameters. In addition, we identified the potential prognostic value of VSIG1 for the post-resection survival of gastric cancer patients.

TABLE I. Correlation Between VSIG1 Expression and Clinicopathological Variables of 232 Gastric Cancer Cases

\begin{tabular}{|c|c|c|c|c|c|}
\hline \multirow[b]{2}{*}{ Clinicopathological parameters } & \multirow[b]{2}{*}{$\mathrm{n}^{\mathrm{a}}$} & \multicolumn{2}{|c|}{ VSIG1 expression } & \multirow[b]{2}{*}{$\chi^{2}$} & \multirow[b]{2}{*}{$P$-value } \\
\hline & & Positive & Negative & & \\
\hline All & 232 & 106 & 126 & & \\
\hline \multicolumn{6}{|l|}{ Age (years) } \\
\hline$<55$ & 121 & 61 & 60 & 2.274 & 0.132 \\
\hline$\geq 55$ & 111 & 45 & 66 & & \\
\hline Gender & & & & 1.739 & 0.187 \\
\hline Female & 73 & 38 & 35 & & \\
\hline Male & 159 & 68 & 91 & & \\
\hline Tumor size $(\mathrm{cm})$ & & & & 7.236 & $0.007^{*}$ \\
\hline$<3$ & 29 & 20 & 9 & & \\
\hline$\geq 3$ & 203 & 86 & 117 & & \\
\hline $\mathrm{T}$ stage & & & & 9.496 & $0.023^{*}$ \\
\hline $\mathrm{T} 1$ & 17 & 13 & 4 & & \\
\hline $\mathrm{T} 2$ & 20 & 12 & 8 & & \\
\hline $\mathrm{T} 3$ & 183 & 76 & 107 & & \\
\hline $\mathrm{T} 4$ & 12 & 5 & 7 & & \\
\hline $\mathrm{N}$ stage & & & & 5.586 & 0.134 \\
\hline No & 60 & 32 & 28 & & \\
\hline N1 & 99 & 45 & 54 & & \\
\hline $\mathrm{N} 2$ & 49 & 23 & 26 & & \\
\hline N3 & 24 & 6 & 18 & & \\
\hline M stage & & & & 4.331 & $0.037^{*}$ \\
\hline M0 & 214 & 102 & 112 & & \\
\hline M1 & 18 & 4 & 14 & & \\
\hline Grade & & & & 0.578 & 0.749 \\
\hline 1 & 4 & 2 & 2 & & \\
\hline 2 & 42 & 17 & 25 & & \\
\hline 3 & 186 & 87 & 99 & & \\
\hline Locus & & & & 7.004 & 0.072 \\
\hline Cardia and fundus & 84 & 32 & 52 & & \\
\hline Corpus & 36 & 19 & 17 & & \\
\hline Antrum & 87 & 47 & 40 & & \\
\hline Other sites ${ }^{b}$ & 25 & 8 & 17 & & \\
\hline
\end{tabular}

VSIG1, V-set and immunoglobulin domain containing 1.

${ }^{a}$ Numbers of cases in each group.

${ }^{\mathrm{b}}$ Mainly remnant gastric cancer.

* Statistically significant $(P<0.05)$. 


\section{MATERIALS AND METHODS}

\section{Patients and Follow-Up}

The clinicopathological data from 232 gastric cancer patients who underwent surgical resection at the Sun Yat-Sen University Cancer Center between 2003 and 2006 were retrospectively analyzed. Patients who met all the following eligibility criteria were included in our study: (1) diagnosis of gastric adenocarcinoma identified by histopathological examination; (2) surgical history that included gastrectomy plus lymphadenectomy (limited or extended); (3) availability of complete follow-up data; (4) no preoperative treatment, such as chemotherapy and radiotherapy; (5) no history of familial malignancy or other synchronous malignancy; (6) no recurrent gastric cancer, and (7) no death during the perioperative period. Tumor resection and D2 lymphadenectomy were performed by experienced surgeons, and the surgical procedure of radical resection, following the Japanese Gastric Cancer Association (JGCA) guidelines [24], was similar in all patients. These patients included 159 males and 73 females, with a median age of 59 years (range: $23-85$ years). Gastric cancer was primarily diagnosed by imaging (X-ray, CT, MRI, etc.), endoscopy, and biopsy. After operation, histopathological diagnosis of each tumor specimen was confirmed with hematoxylin-eosin (HE) staining in the Department of Pathology. The histopathological type and grade were determined using the criteria of the World Health Organization (WHO) classification [25]. All the gastric adenocarcinomas were graded based on their glandular differentiation degree, with Grade 1 for well-differentiated adenocarcinomas (more than $95 \%$ of tumor composed of glands), including tubular adenocarcinomas; Grade 2 for moderately differentiated adenocarcinomas (5095\% of tumor composed of glands), and Grade 3 for poorly differentiated carcinomas (49\% or less of tumor composed of glands). All patients were staged according to the seventh-edition Tumor Node Metastasis (TNM) staging system of the Union for International Cancer Control (UICC) and American Joint Committee on Cancer (AJCC) [26]. Post-operative follow-up, including physical and laboratory examinations, was performed at the outpatient department every 3 months for the first 2 years, every 6 months for the 3rd to 5 th years and annually thereafter until at least 5 years after the operation or until the patient died, whichever came first. The follow-up was closed in September, 2011. The median follow-up for the entire cohort was 57 months (range: 3-91 months). Overall survival (OS), defined as the time from operation to death or last follow-up, was used as a measure of prognosis. Disease-free survival (DFS) is defined as the time elapsed from operation to the date of the recurrence or distant metastasis of gastric cancer (with histopathological confirmation or imaging diagnostic evidence of tumor recurrence or metastasis); development of a second nongastric cancer (with the exception of skin basal cell carcinoma; ductal or lobular breast carcinoma in situ and cervical carcinoma in situ), or death, whichever occurred first. The characteristics of these patients are listed in Table I.

\section{Gastric Cancer Tissues}

For real-time quantitative PCR and Western blotting analyses, a total of 30 paired cancerous tissues and matched adjacent noncancerous gastric mucosa located at least $2 \mathrm{~cm}$ away from the cancer were collected from gastric adenocarcinoma patients undergoing gastrectomy at Sun Yat-Sen University Cancer Center between March and June, 2009. The 30 patients included 19 men and 11 women, with a median age of 53 years (range: 31-77 years). After resection, the fresh tissues were immediately frozen in liquid nitrogen and stored at $-80^{\circ} \mathrm{C}$. Both the cancerous and noncancerous gastric mucosa tissues were verified by histopathological examination. For immunohistochemical staining, formalin-fixed, paraffin-embedded primary

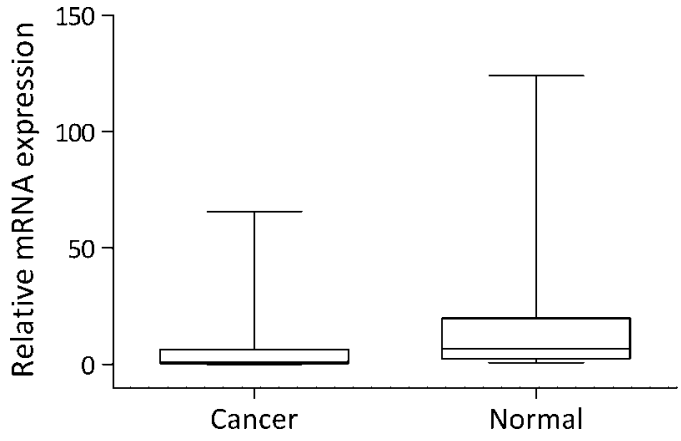

Fig. 1. Decreased VSIG1 mRNA expression in gastric cancer tissues as assessed by real-time quantitative PCR $(\mathrm{n}=30, P=0.021)$.

gastric adenocarcinoma samples were collected from the 232 patients mentioned above and stored at room temperature. HE slides from these patients were viewed under a light microscope by a pathologist and $5-\mu \mathrm{m}$ thick tissue sections were cut from corresponding blocks containing representative tumor regions. The research was approved by the Ethical Committee of Sun Yat-Sen University Cancer Center, and informed consent was obtained from each patient.

\section{Cell Culture}

Gastric cancer cell lines SGC7901, MGC803, HGC27, MKN45, and AGS were obtained from the Committee of Type Culture Collec-

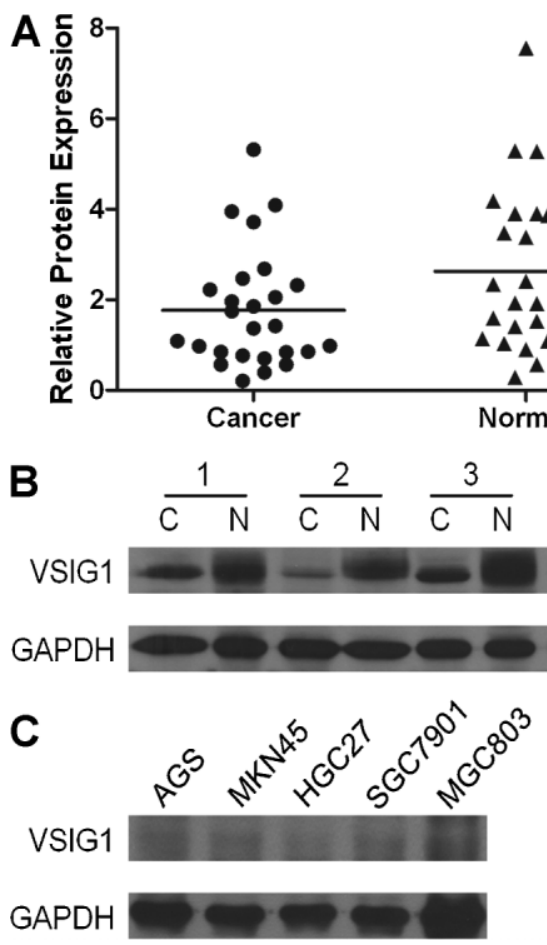

Fig. 2. Decreased VSIG1 protein expression in gastric cancer as assessed by Western blotting. A: Relative VSIG1 protein expression levels in gastric cancer tissues and noncancerous tissues (VSIG1/ GAPDH, $\mathrm{n}=26, P=0.033$ ). Horizontal lines represent the mean. B: Representative VSIG1 protein expression in four paired gastric cancer and noncancerous mucosa $(\mathrm{C}$, gastric cancer tissues; $\mathrm{N}$, matched noncancerous gastric mucosa). C: Loss of VSIG1 expression in five gastric cancer cell lines. 
tion of Chinese Academy of Sciences (Shanghai, China) and cultured in RPMI 1640 medium supplemented with $10 \%$ (v/v) fetal bovine serum at $37^{\circ} \mathrm{C}$ with $5 \% \mathrm{CO}_{2}$.

\section{RNA Extraction and Quantitative Real-Time PCR}

Total RNA was extracted using TRIzol reagent (Invitrogen, Carlsbad, CA) according to the manufacturer's protocol. Then, $2 \mu \mathrm{g}$ of RNA was reverse transcribed into first-strand cDNA by M-MLV Reverse Transcriptase (Promega, Madison, WI) according to the manufacturer's instructions. VSIG1 and GAPDH were amplified by quantitative real-time PCR using the following primers: VSIG1 forward: 5'-GATTGGTAGCCTGGTAGGTGCC-3', reverse: 5'-CCGCGATGGTCTTAGAATTTCT-3'; GAPDH forward: 5'-CTCCTCCTGTTCGACAGTCAGC-3', reverse: 5'-CCCAATACGACCAAATCCGTT-3'. Gene-specific amplification was performed in an ABI 7900HT real-time PCR system (Life Technologies, Carlsbad, CA) with a 15$\mu l$ PCR mix containing $0.5 \mu l$ of cDNA, $7.5 \mu l$ of $2 \times$ SYBR Green master mix (Invitrogen), and $200 \mathrm{nM}$ of the appropriate primers. The mix was preheated at $95^{\circ} \mathrm{C}$ for $10 \mathrm{~min}$ and then amplified in 45 cycles of $95^{\circ} \mathrm{C}$ for $30 \mathrm{sec}$ and $60^{\circ} \mathrm{C}$ for $1 \mathrm{~min}$. The resolution curve was measured at $95^{\circ} \mathrm{C}$ for $15 \mathrm{sec}, 60^{\circ} \mathrm{C}$ for $15 \mathrm{sec}$, and $95^{\circ} \mathrm{C}$ for $15 \mathrm{sec}$. The $\mathrm{C}_{\mathrm{t}}$ (threshold cycle) value of each sample was calculated, and the relative expression of VSIG1 mRNA was normalized to the GAPDH value $\left(2^{-\Delta \mathrm{Ct}}\right.$ method).

\section{Western Blotting Analysis}

Homogenized tissues were lysed in RIPA lysis buffer, and the lysates were harvested by centrifugation $(12,000 \mathrm{rpm})$ at $4{ }^{\circ} \mathrm{C}$ for $30 \mathrm{~min}$. Next, the protein samples $(20 \mu \mathrm{g})$ were separated by electrophoresis in a $12 \%$ SDS-PAGE and were transferred onto a polyvinylidene fluoride membrane. The membrane was placed in $5 \%$ nonfat milk for $1 \mathrm{hr}$ to block the nonspecific binding sites and was then incubated with a sheep anti-human VSIG1 antibody $(1: 1,000$, R\&D Systems, Minneapolis, MN) at $4^{\circ} \mathrm{C}$ overnight. After washing four times in Tris-buffered saline with Tween-20, the membrane was probed with a horseradish peroxidase (HRP)-conjugated rabbit antisheep IgG antibody $(1: 2,000$, Proteintech Group, Chicago, IL) at $37^{\circ} \mathrm{C}$ for $60 \mathrm{~min}$. After four washes, the bands were detected with the enhanced chemiluminescence reagent (Cell Signaling Technology, Danvers, MA). Band density was measured with ImageJ software (National Institutes of Health, Bethesda, MD) and was standardized to that of GAPDH detected using mouse anti-human GAPDH monoclonal antibody (Shanghai Kangchen, Shanghai, China).

\section{Immunohistochemistry}

After deparaffinization with dimethylbenzene, the tissue sections were rehydrated through $100 \%, 95 \%, 90 \%, 80 \%$, and $70 \%$ ethanol After three washes in phosphate-buffered saline (PBS), the slides were boiled in antigen retrieval buffer containing $1 \mathrm{mM}$ of disodium ethylenediaminetetraacetic acid $(\mathrm{pH}=8.0)$ for $15 \mathrm{~min}$ in a microwave oven, and the slides were then rinsed in peroxidase quenching solution (Invitrogen) to block endogenous peroxidase. The sections were then incubated with a sheep anti-human VSIG1 polyclonal antibody (1:200) at $4{ }^{\circ} \mathrm{C}$ overnight and then with an HRP-conjugated rabbit anti-sheep $\operatorname{IgG}$ antibody (1:200) at room temperature for 30 min. Finally, 3,3'-diaminobenzidine (DAB) solution was added to the sections to develop the color, followed by counterstaining with hematoxylin. For negative controls, adjacent sections were processed as described above except that they were incubated overnight at $4{ }^{\circ} \mathrm{C}$ in blocking solution without the primary antibody.

The intensity and extent of VSIG1 immunostaining were evaluated for all samples under double-blinded conditions. In brief, the percentage of positive staining was scored as $0(0-9 \%), 1(10-25 \%), 2$
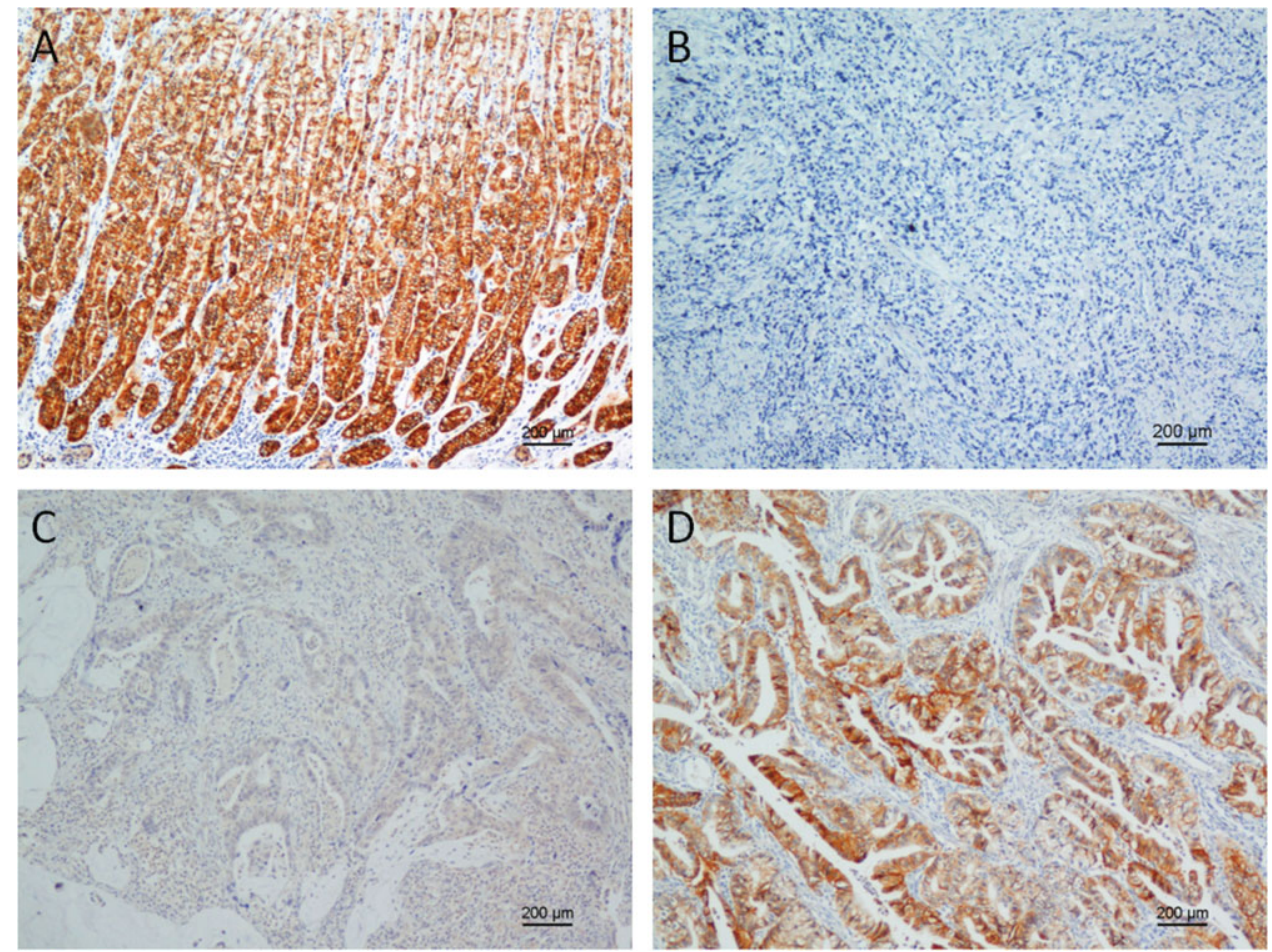

Fig. 3. VSIG1 expression in gastric epithelia and cancer tissues by immunohistochemical staining. A: Strong VSIG1 staining was observed in noncancerous gastric mucosa. B: VSIG1-negative gastric adenocarcinoma, Grade 3, Stage T4N1M0. C: Weak VSIG1 staining in gastric adenocarcinoma, Grade 2, Stage T3N1M0. D: Strong VSIG1 staining in gastric adenocarcinoma, Grade 2, Stage T2N1M0. 
$(26-50 \%)$, or $3(51-100 \%)$, and the intensity as 0 (no staining), 1 (weak staining), 2 (moderate staining), or 3 (dark staining). The total score was calculated as the product of intensity and extent, ranging from 0 to 9. The expression level of VSIG1 was defined as following: "-" (negative, score 0), "+" (weakly positive, score 1-3), “++" (positive, score 4-6), “+++" (strongly positive, score 7-9).

\section{Statistical Analysis}

Differences in mRNA expression between cancerous and normal gastric tissues were evaluated with the Wilcoxon signed ranks test, whereas protein expression levels were compared with paired Student's $t$-test. A chi-squared test was used to analyze the relationships between VSIG1 expression and various clinicopathological parameters. A Kaplan-Meier survival function was calculated and compared with a log-rank test. A Cox proportional hazard regression model was used for univariate and multivariate analyses to explore the effects of the clinicopathological variables and VSIG1 expression on survival. SPSS 17.0 software (SPSS, Chicago, IL) was used for all statistical analyses, and $P<0.05$ was considered significant.

\section{RESULTS VSIG1 mRNA Expression}

We first measured VSIG1 mRNA levels in 30 paired cancerous and normal gastric tissues using quantitative real-time PCR. As shown in Figure 1, the median VSIG1 mRNA expression level was significantly lower in gastric cancer tissues than the corresponding normal gastric tissues $(P=0.021)$, and $76 \%$ of the subjects $(23 / 30)$ displayed lower VSIG1 mRNA expression in cancer tissues.

\section{VSIG1 Protein Expression}

We then determined VSIG1 protein levels in resected gastric cancer samples and cell lines by Western blotting. Consistent with the quantitative real-time PCR results, out of a total of 26 paired cancerous and adjacent normal tissues, VSIG1 expression was reduced in cancerous tissues relative to their respective adjacent normal tissues in 18 cases $(P=0.033$, Fig. 2A,B). No VSIG1 expression was detected in the 5 gastric cancer cell lines (Fig. 2C).

\section{Immunohistochemical Analysis of VSIG1 Expression in Gastric Cancer and Its Relationship With the Clinicopathological Parameters}

To validate the above findings and investigate the clinicopathological and prognostic roles of VSIG1 expression, we performed immunohistochemical analyses of the 232 paraffin-embedded gastric cancer tissue blocks. Overall, 126 of 232 (54.3\%) cases showed negative VSIG1 expression in cancerous tissues (Fig. 3B), whereas $106(45.7 \%)$ cases showed positive immunostaining (Fig. 3C,D). Normal gastric mucosa showed the strongest VSIG1 positive staining (Fig. 3A). As listed in Table I, the loss of VSIG1 expression was significantly correlated with tumor size $(P=0.007)$, depth of tumor infiltration (T stage, $P=0.023$ ), and distant metastasis (M stage, $P=0.037$ ), but not with age, gender, tumor locus, or local lymph node metastasis ( $\mathrm{N}$ stage).

\section{VSIG1 Expression and clinical Outcome}

As shown in Figure 4, patients with VSIG1-negative gastric cancer showed shorter OS $(P<0.001$, log-rank test, Fig. 4A) and
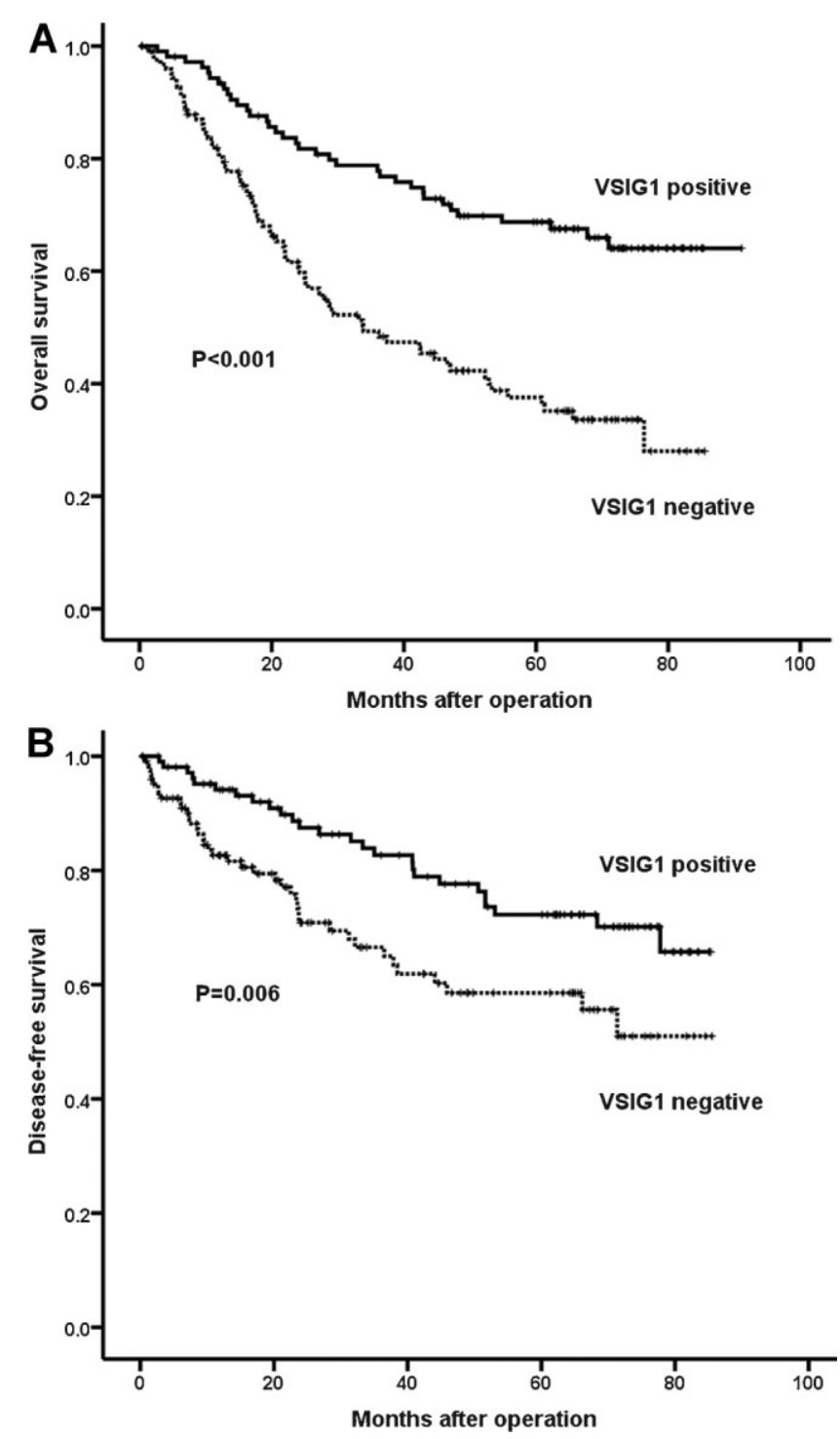

Fig. 4. Kaplan-Meier survival curves of gastric cancer patients $(\mathrm{n}=232)$ after gastrectomy. Patients with VSIG1-negative gastric cancer showed both significantly worse OS (A) and DFS (B) than those with VSIG1-positive gastric cancer (log-rank test, $P<0.001$ and $P=0.006$, respectively).

poorer DFS $(P=0.006$, log-rank test, Fig. $4 \mathrm{~B})$ than those with VSIG1-positive gastric cancer. Univariate Cox regression analyses revealed that OS significantly decreased with larger tumor size $(P=0.009)$, higher $\mathrm{T}$ stage $(P<0.001)$, higher $\mathrm{N}$ stage $(P<0.001)$, higher $\mathrm{M}$ stage $(P<0.001)$, and negative VSIG1 expression $(P<0.001)$, while DFS significantly decreased with higher T stage $(P=0.047)$, higher $\mathrm{N}$ stage $(P=0.016)$, higher $\mathrm{M}$ stage $(P=0.035)$, and negative VSIG1 expression $(P=0.007$; Table II $)$. Furthermore, multivariate Cox regression analyses confirmed T stage $(P=0.023), \mathrm{N}$ stage $(P<0.001), \mathrm{M}$ stage $(P<0.001)$, and VSIG1 expression $(P=0.002)$ as significant independent predictors of the OS of gastric cancer patients, whereas $M$ stage $(P=0.032)$ and VSIG1 expression $(P=0.039)$ were significant independent prognostic factors for DFS (Table III). 
VSIG1 Expression in Gastric Cancer

TABLE II. Univariate Analyses of Overall and Disease-Free Survival of Gastric Cancer Patients

\begin{tabular}{|c|c|c|c|c|c|c|c|}
\hline \multirow[b]{2}{*}{ Variables } & \multirow[b]{2}{*}{$\mathrm{n}^{\mathrm{a}}$} & \multicolumn{3}{|c|}{ Overall survival } & \multicolumn{3}{|c|}{ Disease-free survival } \\
\hline & & $\mathrm{HR}$ & $95 \% \mathrm{CI}$ & $P$-value & HR & $95 \% \mathrm{CI}$ & $P$-value \\
\hline Age & & & & 0.144 & & & 0.194 \\
\hline$<55$ & 121 & 1.000 & & & 1.000 & & \\
\hline$\geq 55$ & 111 & 1.326 & $0.908-1.935$ & & 0.721 & $0.440-1.182$ & \\
\hline Gender & & & & 0.748 & & & 0.235 \\
\hline Female & 73 & 1.000 & & & 1.000 & & \\
\hline Male & 159 & 0.936 & $0.625-1.401$ & & 0.738 & $0.446-1.219$ & \\
\hline Tumor size & & & & $0.009^{*}$ & & & 0.282 \\
\hline$<3 \mathrm{~cm}$ & 29 & 1.000 & & & 1.000 & & \\
\hline$\geq 3 \mathrm{~cm}$ & 203 & 3.000 & $1.316-6.840$ & & 1.539 & $0.702-3.374$ & \\
\hline $\mathrm{T}$ stage & & & & $<0.001^{*}$ & & & $0.047^{*}$ \\
\hline $\mathrm{T} 1$ & 17 & 1.000 & & & 1.000 & & \\
\hline $\mathrm{T} 2$ & 20 & 2.012 & $0.182-22.188$ & & 3.575 & $0.372-34.394$ & \\
\hline $\mathrm{T} 3$ & 183 & 13.406 & $1.868-96.197$ & & 9.023 & $1.248-65.258$ & \\
\hline $\mathrm{T} 4$ & 12 & 28.014 & $3.494-224.629$ & & 13.979 & $1.554-125.759$ & \\
\hline $\mathrm{N}$ stage & & & & $<0.001^{*}$ & & & $0.016^{*}$ \\
\hline No & 60 & 1.000 & & & 1.000 & & \\
\hline $\mathrm{N} 1$ & 99 & 3.347 & $1.737-6.449$ & & 2.083 & $1.043-4.160$ & \\
\hline $\mathrm{N} 2$ & 49 & 5.518 & $2.768-11.000$ & & 3.146 & $1.481-6.685$ & \\
\hline N3 & 24 & 7.972 & $3.743-16.981$ & & 3.365 & $1.292-8.761$ & \\
\hline M stage & & & & $<0.001^{*}$ & & & $0.035^{*}$ \\
\hline M0 & 214 & 1.000 & & & 1.000 & & \\
\hline M1 & 18 & 6.514 & $3.882-10.929$ & & 2.725 & $1.074-6.911$ & \\
\hline Grade & & & & 0.537 & & & 0.977 \\
\hline 1 & 4 & 1.000 & & & 1.000 & & \\
\hline 2 & 42 & 6770.916 & $0-7.858 \times 10^{48}$ & & 8270.475 & $0-3.458 \times 10^{58}$ & \\
\hline 3 & 186 & 9059.141 & $0-1.050 \times 10^{49}$ & & 8706.358 & $0-3.637 \times 10^{58}$ & \\
\hline Locus & & & & 0.093 & & & 0.330 \\
\hline Cardia and fundus & 84 & 1.000 & & & 1.000 & & \\
\hline Corpus & 36 & 0.854 & $0.501-1.455$ & & 0.855 & $0.410-1.782$ & \\
\hline Antrum & 87 & 0.563 & $0.358-0.887$ & & 0.727 & $0.409-1.290$ & \\
\hline Other sites ${ }^{\mathrm{b}}$ & 25 & 0.928 & $0.481-1.790$ & & 1.474 & $0.688-3.160$ & \\
\hline VSIG1 & & & & $<0.001^{*}$ & & & $0.007^{*}$ \\
\hline Negative & 126 & 1.000 & & & 1.000 & & \\
\hline Positive & 106 & 0.378 & $0.252-0.568$ & & 0.505 & $0.307-0.832$ & \\
\hline
\end{tabular}

HR, hazard ratio; CI, confidence interval; VSIG1, V-set and immunoglobulin domain containing 1.

${ }^{\mathrm{a}}$ Numbers of cases in each group.

${ }^{\mathrm{b}}$ Mainly remnant gastric cancer.

${ }^{*}$ Statistically significant $(P<0.05)$.

\section{DISCUSSION}

Gastric cancer remains one of the most deadly human malignancies. Even with advances in diagnosis and therapy, the prognosis for gastric cancer is still dismal [27]. The clinical outcome of gastric cancer is determined by local tumor growth, invasion, and distant metastasis [3], all of which involve dysfunctional cell adhesion [7]. Although many previous studies have addressed the reorganization of certain adherent junction proteins, such as the E-cadherin/ $\beta$-catenin complex [8], the roles of tight junctions in gastric cancer progression are only now beginning to be revealed. Tight junction proteins are now considered active regulators of cell proliferation, differentiation, migration, and gene transcription [12].

JAMs are type I tight junction proteins characterized by two extracellular immunoglobulin (Ig)-like folds with intramolecular disulfide bonds, a single transmembrane domain, and a cytoplasmic domain containing a PDZ-binding motif [12]. These proteins are classified as $\mathrm{C} 1, \mathrm{C} 2, \mathrm{~V}$, and I types based on their similarity to the constant and variable Ig regions. Although members of this protein family have been previously studied in several kinds of malignancies
$[13,14]$, the expression and roles of JAMs in gastric cancer are rarely investigated, with the exception of CAR, which is expressed in all the examined normal gastric mucosa samples (175/175), but silenced in 87 out of the $196(44.4 \%)$ gastric cancer samples [21]. In addition, the expression of CAR is completely lost in KATO III and MKN38 gastric cell lines and reduced obviously in the MKN45 cell line, whereas AGS cell line shows robust CAR expression. In this study, we examined the mRNA and protein expression levels of VSIG1, a $\mathrm{V}$ type JAM member, in paired gastric cancer tissues and nonmalignant gastric mucosa. Consistent with the findings of Scanlan et al. [22] and the microarray study of Junnila et al. [23], we found that both the mRNA and protein levels of VSIG1 were significantly lower in cancerous tissues than the respective noncancerous mucosa. In addition, no VSIG1 expression was detected in five gastric cancer cell lines.

To further validate this reduction of VSIG1 expression in gastric cancer, we performed an immunohistochemical analysis with a sheep anti-hVSIG1 antibody. In agreement with the Western blotting results, more than $50 \%$ of the cancer samples displayed negative VISG1 immunostaining. Furthermore, among the VSIG1positive cases, most showed lower VSIG1 immunostaining relative 
TABLE III. Multivariate Analyses of Overall and Disease-Free Survival of Gastric Cancer Patients

\begin{tabular}{|c|c|c|c|c|c|c|c|}
\hline \multirow[b]{2}{*}{ Variables } & \multirow[b]{2}{*}{$\mathrm{n}^{\mathrm{a}}$} & \multicolumn{3}{|c|}{ Overall survival } & \multicolumn{3}{|c|}{ Disease-free survival } \\
\hline & & HR & $95 \% \mathrm{CI}$ & $P$-value & HR & $95 \% \mathrm{CI}$ & $P$-value \\
\hline Tumor size & & & & 0.746 & & & \\
\hline$<3 \mathrm{~cm}$ & 29 & 1.000 & & & & & \\
\hline$\geq 3 \mathrm{~cm}$ & 203 & 1.158 & $0.447-2.810$ & & & & \\
\hline $\mathrm{T}$ stage & & & & $0.023^{*}$ & & & 0.206 \\
\hline $\mathrm{T} 1$ & 17 & 1.000 & & & 1.000 & & \\
\hline $\mathrm{T} 2$ & 20 & 2.514 & $0.207-30.475$ & & 3.506 & $0.358-34.380$ & \\
\hline $\mathrm{T} 3$ & 183 & 9.456 & $1.147-77.978$ & & 6.431 & $0.861-48.031$ & \\
\hline $\mathrm{T} 4$ & 12 & 15.456 & $1.697-140.764$ & & 8.679 & $0.925-81.452$ & \\
\hline $\mathrm{N}$ stage & & & & $<0.001^{*}$ & & & 0.134 \\
\hline No & 60 & 1.000 & & & 1.000 & & \\
\hline $\mathrm{N} 1$ & 99 & 2.999 & $1.544-5.826$ & & 1.744 & $0.863-3.524$ & \\
\hline $\mathrm{N} 2$ & 49 & 5.214 & $2.560-10.622$ & & 2.506 & $1.159-5.418$ & \\
\hline N3 & 24 & 7.737 & $2.626-12.533$ & & 2.068 & $0.768-5.514$ & \\
\hline M stage & & & & $<0.001^{*}$ & & & $0.032^{*}$ \\
\hline Mo & 214 & 1.000 & & & 1.000 & & \\
\hline M1 & 18 & 7.581 & $4.293-13.386$ & & 2.917 & $1.096-7.765$ & \\
\hline VSIG1 & & & & $0.002^{*}$ & & & $0.039^{*}$ \\
\hline Negative & 126 & 1.000 & & & 1.000 & & \\
\hline Positive & 106 & 0.507 & $0.3270-.0786$ & & 0.581 & $0.347-0.973$ & \\
\hline
\end{tabular}

HR, hazard ratio; CI, confidence interval; VSIG1, V-set and immunoglobulin domain containing 1.

${ }^{\mathrm{a}}$ Numbers of cases in each group.

* Statistically significant $(P<0.05)$.

to the noncancerous mucosa. Scanlan et al. [22] previously reported a positive VSIG1 immunostaining rate of only $29 \%$ in gastric cancer samples (5/17). This discrepancy may stem from the difference in sample size, as our study assessed a much larger group of patients (232 cases).

We also found that the loss of VSIG1 expression was associated with higher $\mathrm{T}$ stage and larger tumor size, implying that the absence of VSIG1 might promote tumor growth and invasion. In addition, we detected lower VSIG1 immunostaining in stage M1 compared with M0 gastric cancer tissues, suggesting that decreased VSIG1 expression may facilitate tumor metastasis. These findings collectively indicate an important role for VSIG1 in the growth and metastasis of gastric cancer. Whether the loss of VSIG1 is a cause or consequence of tumor progression and metastasis, however, remains unclear. Considering that VSIG1 is a cell adhesion protein of the JAM family, our data support the concept that disrupted intercellular adhesion is a prerequisite for tumor growth and metastasis, in accordance with the role described for E-cadherin in gastric cancer $[28,29]$. Indeed, other JAM family members have been shown to function as tumor suppressors [15]. Anders et al. [21] found that enforced ectopic expression of CAR inhibited the proliferation, migration, and invasion of gastric cell lines, whereas RNAi-mediated CAR knockdown had opposite effects. Collectively, these data have suggested important biological roles of JAMs in gastric carcinogenesis.

Kaplan-Meier survival analyses revealed a significant correlation between the loss of VSIG1 expression and poor survival of gastric cancer patients after gastrectomy. A Cox regression model further demonstrated that the VSIG1 expression level was an independent risk factor for both OS and DFS, suggesting that VSIG1 may serve as a prognostic biomarker for gastric cancer patients after surgery. These data are in accordance with the concept of the correlation between disturbed cell adhesion and poor prognosis in gastric cancer [30]. In particular, it is widely accepted that the loss of E-cadherin expression is associated with unfavorable survival in gastric cancer patients [29]. However, only a few studies have investigated the association between the expression of tight junction proteins, especially the JAM family proteins, and gastric cancer prognosis. Two previous studies reported that high expression of either claudin4 or claudin-18 was significantly correlated with a better prognosis $[31,32]$. For JAM family members, only one report to date has correlated CAR expression with the gastric cancer patient survival [21]. Our finding that the loss of VSIG1 expression in gastric cancer is associated with more malignant phenotypes and a worse prognosis suggests that this tight junction protein may play a tumor suppressor role in gastric cancer cells.

VSIG1 expression is enriched in the testis, and it was reported that the Ig domain of VSIG1 interacts with Sertoli cell membrane protein [33]. The VSIG1 molecule belongs to the JAM family, which contains two Ig-like domains in their extracellular area [34]. The roles of other members of this family have been studied in breast cancer and renal cancer $[13,14]$. In gastric cancer, CAR, a member of the JAM family, has been shown to repress the proliferation, migration, and invasion of gastric cancer cells [21]. Nevertheless, there is no report concerning the prognostic roles of VSIG1 in cancer prior to this study. Considering the structural similarity and sequence homology of this protein family, it is possible that VSIG1 may suppress the gastric carcinogenesis in a similar manner as CAR and JAM-A. While our study has demonstrated a correlation between VSIG1 expression and gastric cancer stage and prognosis, the mechanism involved in biological function of VSIG1 in gastric cancer warranties further investigation.

\section{CONCLUSIONS}

In the present study, we report the loss of VSIG1 expression in gastric cancers and show that the absence of this protein is correlated with a more malignant phenotype and a worse clinical outcome. Our findings contribute to the current understanding on the role of tight junctions in gastric cancer. To our best knowledge, the data generated in this study represent the first report correlating the presence of VSIG1 with clinicopathological characteristics as well as with the OS and DFS of gastric cancer patients. 


\section{ACKNOWLEDGMENTS}

We thank Professor Zhou Zhiwei for his kind help in collecting gastric cancer samples. This work was supported by National Natural Foundation of China (No. 30973398), and was partially supported by the Gillson Longenbaugh Foundation.

\section{REFERENCES}

1. Jemal A, Bray F, Center MM, et al.: Global cancer statistics. CA Cancer J Clin 2011;61:69-90.

2. Polk DB, Peek RM, Jr.: Helicobacter pylori: Gastric cancer and beyond. Nat Rev Cancer 2010;10:403-414.

3. Ushijima T, Sasako M: Focus on gastric cancer. Cancer Cell 2004:5:121-125.

4. Tamura G: Alterations of tumor suppressor and tumor-related genes in the development and progression of gastric cancer. World J Gastroenterol 2006;12:192-198.

5. Takeno A, Takemasa I, Doki Y, et al.: Integrative approach for differentially overexpressed genes in gastric cancer by combining large-scale gene expression profiling and network analysis. Br J Cancer 2008;99:1307-1315.

6. Myllykangas S, Junnila S, Kokkola A, et al.: Integrated gene copy number and expression microarray analysis of gastric cancer highlights potential target genes. Int J Cancer 2008;123: 817-825.

7. Makrilia N, Kollias A, Manolopoulos L, et al.: Cell adhesion molecules: Role and clinical significance in cancer. Cancer Invest 2009;27:1023-1037.

8. Guilford P, Hopkins J, Harraway J, et al.: E-cadherin germline mutations in familial gastric cancer. Nature 1998;392:402-405.

9. Perl AK, Wilgenbus P, Dahl U, et al.: A causal role for Ecadherin in the transition from adenoma to carcinoma. Nature 1998;392:190-193.

10. Cavallaro U, Christofori G: Cell adhesion and signalling by cadherins and Ig-CAMs in cancer. Nat Rev Cancer 2004;4:118132.

11. Li SG, Ye ZY, Zhao ZS, et al.: Correlation of integrin beta3 mRNA and vascular endothelial growth factor protein expression profiles with the clinicopathological features and prognosis of gastric carcinoma. World J Gastroenterol 2008;14:421-427.

12. Gonzalez-Mariscal L, Lechuga S, Garay E: Role of tight junctions in cell proliferation and cancer. Prog Histochem Cytochem 2007;42:1-57.

13. Gutwein P, Schramme A, Voss B, et al.: Downregulation of junctional adhesion molecule-A is involved in the progression of clear cell renal cell carcinoma. Biochem Biophys Res Commun 2009;380:387-391.

14. Naik MU, Naik TU, Suckow AT, et al.: Attenuation of junctional adhesion molecule-A is a contributing factor for breast cancer cell invasion. Cancer Res 2008;68:2194-2203.

15. Lamagna C, Hodivala-Dilke KM, Imhof BA, et al.: Antibody against junctional adhesion molecule-C inhibits angiogenesis and tumor growth. Cancer Res 2005;65:5703-5710.

16. Jung $\mathrm{H}$, Jun $\mathrm{KH}$, Jung JH, et al.: The expression of claudin-1, claudin-2, claudin-3, and claudin-4 in gastric cancer tissue. J Surg Res 2011;167:e185-191.
17. Chang TL, Ito K, Ko TK, et al.: Claudin-1 has tumor suppressive activity and is a direct target of RUNX3 in gastric epithelial cells. Gastroenterology 2010;138:255-265, e251-253.

18. Agarwal R, Mori Y, Cheng Y, et al.: Silencing of claudin-11 is associated with increased invasiveness of gastric cancer cells. PLoS ONE 2009;4:e8002.

19. Park JY, Park KH, Oh TY, et al.: Up-regulated claudin 7 expression in intestinal-type gastric carcinoma. Oncol Rep 2007;18: 377-382.

20. Oshima T, Miwa H, Joh T: Aspirin induces gastric epithelial barrier dysfunction by activating p38 MAPK via claudin-7. Am J Physiol Cell Physiol 2008;295:C800-C806.

21. Anders M, Vieth M, Rocken C, et al.: Loss of the Coxsackie and adenovirus receptor contributes to gastric cancer progression. Br J Cancer 2009;100:352-359.

22. Scanlan MJ, Ritter G, Yin BW, et al.: Glycoprotein A34, a novel target for antibody-based cancer immunotherapy. Cancer Immun 2006;6:2

23. Junnila S, Kokkola A, Mizuguchi T, et al.: Gene expression analysis identifies over-expression of CXCL1, SPARC, SPP1, and SULF1 in gastric cancer. Genes Chromosomes Cancer 2010;49:28-39.

24. Shimada Y: JGCA (The Japan Gastric Cancer Association) Gastric cancer treatment guidelines. Jpn J Clin Oncol 2004; 34:58.

25. Hamilton SR, Aaltonen LA, editors. WHO classification of tumours. Tumours of the digestive system. Lyon: IARC Press; 2000.

26. Sobin LH, Gospodarowicz MK, Wikkekind C, editors. TNM classification of malignant tumours. Hoboken: Wiley-Blackwell; 2009.

27. Hartgrink HH, Jansen EP, van Grieken NC, et al.: Gastric cancer. Lancet 2009:374:477-490.

28. Yonemura Y, Endou Y, Kimura K, et al.: Inverse expression of S100A4 and E-cadherin is associated with metastatic potential in gastric cancer. Clin Cancer Res 2000;6:4234-4242.

29. Yonemura Y, Ninomiya I, Kaji M, et al.: Decreased E-cadherin expression correlates with poor survival in patients with gastric cancer. Anal Cell Pathol 1995;8:177-190.

30. Yasui W, Oue N, Aung PP, et al.: Molecular-pathological prognostic factors of gastric cancer: A review. Gastric Cancer 2005, 8:86-94.

31. Ohtani S, Terashima M, Satoh J, et al.: Expression of tight-junction-associated proteins in human gastric cancer: Downregulation of claudin- 4 correlates with tumor aggressiveness and survival. Gastric Cancer 2009;12:43-51.

32. Sanada Y, Oue N, Mitani Y, et al.: Down-regulation of the claudin-18 gene, identified through serial analysis of gene expression data analysis, in gastric cancer with an intestinal phenotype. J Pathol 2006;208:633-642

33. Kim E, Lee Y, Kim JS, et al.: Extracellular domain of V-set and immunoglobulin domain containing 1 (VSIG1) interacts with sertoli cell membrane protein, while its PDZ-binding motif forms a complex with ZO-1. Mol Cells 2010;30:443-448.

34. Ebnet K, Suzuki A, Ohno S, et al.: Junctional adhesion molecules (JAMs): More molecules with dual functions? J Cell Sci 2004:117:19-29. 